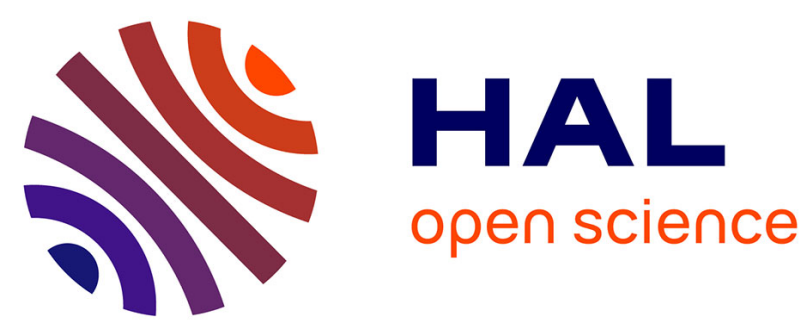

\title{
Analysis and Design of a Hybrid Optical Fiber Refractometer for Large Dynamic Range Measurements
}

Haris Apriyanto, Olivier D Bernal, Michel Cattoen, Françoise Lizion, James Sharp, Frédéric Surre, Valérie Chavagnac, Han Cheng Seat

\section{- To cite this version:}

Haris Apriyanto, Olivier D Bernal, Michel Cattoen, Françoise Lizion, James Sharp, et al.. Analysis and Design of a Hybrid Optical Fiber Refractometer for Large Dynamic Range Measurements. IEEE Sensors Journal, 2020, 20 (10), pp.5260-5269. 10.1109/JSEN.2020.2969417 hal-02568055

\section{HAL Id: hal-02568055 \\ https://hal.laas.fr/hal-02568055}

Submitted on 8 May 2020

HAL is a multi-disciplinary open access archive for the deposit and dissemination of scientific research documents, whether they are published or not. The documents may come from teaching and research institutions in France or abroad, or from public or private research centers.
L'archive ouverte pluridisciplinaire HAL, est destinée au dépôt et à la diffusion de documents scientifiques de niveau recherche, publiés ou non, émanant des établissements d'enseignement et de recherche français ou étrangers, des laboratoires publics ou privés. 


\title{
Analysis and design of a hybrid optical fiber refractometer for large dynamic range measurements
}

\author{
Haris Apriyanto ${ }^{1,2}$, Olivier D. Bernal ${ }^{1}$, Member, IEEE, Michel Cattoen ${ }^{1}$, Françoise Lizion ${ }^{1}$, \\ James $\mathrm{H}^{3}$. Sharp, Frederic Surre ${ }^{4}$, SeniorMember, IEEE, Valérie Chavagnac ${ }^{5}$ and \\ Han Cheng Seat ${ }^{1}$, Member, IEEE \\ ${ }^{1}$ LAAS-CNRS, Université de Toulouse, CNRS, INP \\ ${ }^{2}$ Politeknik Negeri Indramayu, Indramayu, Indonesia \\ ${ }^{3}$ Systems, Power and Energy Research Division, School of Engineering, University of Glasgow \\ ${ }^{4}$ School of Mathematics, Computer Science and Engineering, City, University of London, London \\ ${ }^{5}$ GET-UMR5563, OMP, Université de Toulouse, CNRS, IRD, Toulouse, France
}

DOI: $10.1109 /$ JSEN.2020.2969417

\begin{abstract}
A fiber refractometer with large dynamic range from 1.316 to 1.61 RIU has been realized using a hybrid configuration of a single-mode fiber (SMF) coupled to a stripped-cladding multimode fiber (MMF) as sensing element. An extended analysis of the diffraction principle of a Gaussian beam is specifically developed for this sensor configuration to determine the injected power density into the MMF which, when subsequently combined with ray optics, analytical wave optics and Fresnel equations, enables the sensor response to be comprehensively estimated. Simulation results have been experimentally corroborated to very high agreement for a $2-\mathrm{cm}$ and a $5-\mathrm{cm}$ decladded section of multimode fiber used as the sensing element. The results show, for the shorter sensor $(2 \mathrm{~cm})$, a very high sensitivity of $\sim-250$ a.u./RIU being achieved in the Zone II operating regime, i.e. for indices between the cladding and core indices together with a resolution of $2.76 \times 10^{-6} \mathrm{RIU}$ being attained. In addition, the developed models have been used to accurately predict the response of sensing elements of various lengths, hence demonstrating the potential capability of this research to be exploited for optimizing bespoke design of fiber refractometers of any arbitrary sensing lengths or dimensions. As an example, we present the design of a refractometer achieving a maximum sensitivity of 300 a.u./RIU with a potential resolution of $2.26 \times 10^{-6} \mathrm{RIU}$.
\end{abstract}

Index Terms-Fresnel equation, Gaussian beam, ray optics, wave optics, hybrid sensor design, modelling, multimode fiber, single-mode fiber, fiber refractometer, refractive index measurement.

\section{INTRODUCTION}

A LARGE variety of refractive index (RI) sensors or refractometers exploiting different measurement techniques have been reported since the end of the 19th century [1] due to their broad applications in chemistry and physics, in environmental monitoring, as well as across the biochemical, pharmaceutical, and the food and beverage industries [2].
Amongst these RI sensors, a multitude of optical fiber refractometers (OFRs) have also been developed for their intrinsic advantages, such as their robustness to relatively harsh ambient conditions, explosion-free security since they require no on-probe electrical power, rapid real-time in situ measurement for remote sensing, multiplexing potential, and immunity to electromagnetic interference (EMI) [3-5], to name a few.

Interferometric refractometers have recently been reported with suitable capabilities for RI measurement. A hybrid device exploiting a capillary fiber sandwiched between two SMFs in a Fabry-Perot configuration and interrogated by a swept wavelength laser has been reported for wide RI sensing from $1.3924-1.5882$ RIU with a precision of $7 \times 10^{-4}$ RIU [6]. Fukano et al. reported on a refractometer employing multimode interference in a hybrid sensor comprising an MMF sandwiched between two SMFs with detection performed using an optical spectrum analyzer [7]. Another refractometric technique that is frequently used is that based on surface plasmon resonance, in particular, for biosensing [8].

Nevertheless, one RI measurement method which is widely exploited is the optical intensity-based refractometer [9-25] due to its simplicity and cost effectiveness. In such a scheme, a variation in the RI value will induce a variation in the optical power or intensity to be measured. OFR techniques based on optical intensity modulation have been realized using tapered MMFs [9-11], back-reflection fiber core tips [12-14], dual MMF set-ups similar to the fiber bundle proximity sensor $[15,16]$, D-shaped multimode plastic optical fibers $[17,18]$, and stripped-cladding MMFs [19-25].

Development of the stripped-cladding MMF refractometer has been reported since the 1980's [19-25], inspired by the dielectric rod refractometer immersed in a liquid used as the equivalent cladding [26-28]. Various models of stripped- 


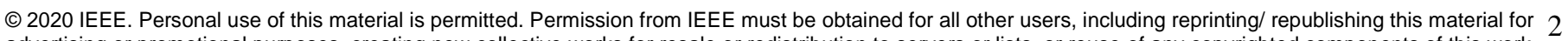
advertising or promotional purposes, creating new collective works for resale or redistribution to servers or lists, or reuse of any copyrighted components of this work in other works.

cladding MMF refractometer have been proposed [19-22] to estimate its optical power response measured against RI variation with respect to the core index. In these early refractometers, Snell's law and Fresnel's reflection law were employed to interpret the sensor response for RIs lower than that of the core, while only Fresnel reflections were employed when the indices were higher than the core index. Although previous models [20,21] attempted to determine and account for the power distribution density of the incident beam, the influence of evanescent wave absorption (EWA) was, however, not considered. Nevertheless, EWA exists since the beams or rays are essentially guided by total internal reflection (TIR) and will have to be accounted for to design an accurate fiber refractometer. In addition, the resulting approximation from these existing models was sufficiently precise only when applied to a beam launched through a lens system [20,21].

We shall here build on an alternative Gaussian beam analysis method previously proposed [25] in order to accurately estimate the optical power distribution density injected from a singlemode (SM) laser beam into an MMF that is used as the sensing element. Although experimentally validated for beam injection via a microscope objective (MO), this method can also be extended to account for any launch conditions, including that from a lead-in SMF spliced to an MMF. Using this improved model subsequently allows the analysis of the impact of both launch conditions and sensing length on the sensor sensitivity in each of the three previously identified RI zones.

As a result, a compact hybrid all-fiber set-up is proposed using an SMF and MMF combination without the inconvenience of opto-mechanical elements which could introduce mechanical perturbations to the sensor as in [25].

\section{OPERATING PRINCIPLE}

\section{A. Determination of power input into the $M M F$}

An SMF is fusion-spliced to a plastic-clad silica MMF (PCS MMF) with a core radius $\left(a_{2}\right)$ of $100 \mu \mathrm{m}$ and a numerical aperture $\left(\mathrm{NA}_{2}\right)$ of 0.50 , as shown in Fig. 1(a). The subsequent lightwave will then be diffracted according to the principles illustrated by Fig. 1(b).

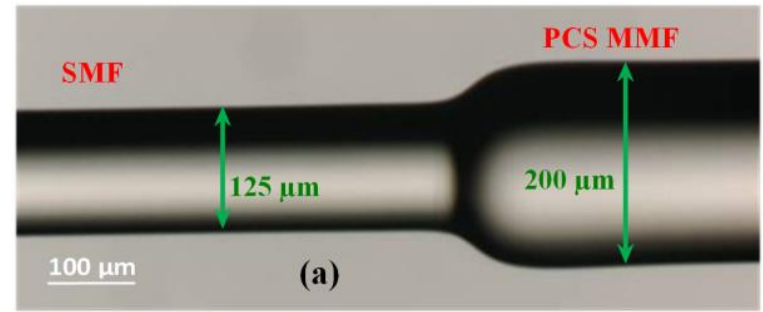

\section{Direction of}

propagation

PCS MMF

SMF

$\stackrel{\boldsymbol{\beta}_{b \downarrow}}{\longrightarrow}$ splicing region between SMF and MMF, and (b) illustration of the principles of lightwave injection and diffraction at the interface of both fibers. Incoming single-mode laser beam is injected through lead-in SMF with radiated beam angle $\beta_{b}$ into MMF at incident beam angle $\theta_{b}$.
Here, it is supposed that the guided beam output from the SMF has similar characteristics to a collimated beam exhibiting a Gaussian distribution. The SMF's mode field diameter $\left(\mathrm{MFD}_{\mathrm{SMF}}\right)$ is then defined as being at $1 / \mathrm{e}^{2}$ of the beam intensity

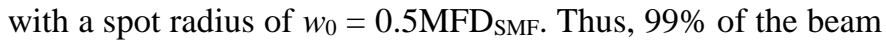
intensity in the SMF is contained within a diameter $D_{0}=\pi w_{0}$ [29]. Furthermore, since the laser beam is launched from the lead-in SMF into the MMF with incident beam angle $\theta_{b}=90^{\circ}$ $\beta_{b}$ (see Fig. 1(b)), $\beta_{b}$ can be calculated in terms of both the SMF's numerical aperture $\left(N A_{1}\right)$ and the core index of the MMF $\left(n_{c o 2}\right)$ as

$$
\beta_{b}=\sin ^{-1}\left(\frac{N A_{1}}{n_{c o 2}}\right)
$$

This Gaussian beam is then diffracted into the MMF until distance $z_{i}$ [30] (see Fig. 1(b)) and will first be incident at the core-cladding interface before being guided in its core. Hence, the true optical power distribution propagated along the MMF should then originate from the power distribution at $z_{i}$. Consequently, according to the principle previously reported [25], the diffraction from the SMF to the MMF core at distance $z_{i}$ for a given operating wavelength $\lambda$ will result in a beam spot radius $\left(w_{z}\right)$ of [31]

$$
w_{z}\left(z_{i}\right)=w_{0} \sqrt{1+\left(\frac{\lambda z_{i}}{n_{c o 2} \pi w_{0}^{2}}\right)^{2}}
$$

where distance $z_{i}$ can be obtained using a trigonometric function in terms of $a_{2}$ and $\beta_{b}$ in the form

$$
z_{i}=a_{2} \cot \beta_{b}
$$

The optical intensity distribution of this Gaussian beam in the MMF at distance $z_{i}\left(I_{z}\right)$ can then be expressed by [31]

$$
I_{z}(a)=I_{0} e^{\left(-\frac{2 a^{2}}{w_{z}^{2}}\right)}
$$

Here, $a$ varies from the center axis of the fiber to its outer radius $a_{2}$ due to the symmetry of revolution. Further, by integrating the optical intensity in (4) over the transverse plane of the beam, followed by normalizing by $I_{0}=\pi w_{z}^{2} / 2$, the normalized input optical power into the MMF can be obtained as $[29,31]$

$$
P_{z}(a)=1-e^{\left(\frac{-2 a^{2}}{w_{z}^{2}}\right)}
$$

The input beam is thus injected from the SMF with $N A_{1}=$ 0.16 at $\lambda=1550 \mathrm{~nm}$ into the $\operatorname{MMF}\left(N A_{2}=0.50\right)$ with its middle section stripped of the cladding or buffer layer. The incident beam angle $\left(\theta_{b}\right)$ in the MMF is thus always higher than its critical angle $\left(\theta_{c}\right)$. Therefore, this beam will be entirely propagated by TIR without any part of it being refracted into the cladding.

\section{B. Sensing mechanisms}

As previously reported [24,25], the sensing mechanisms in the stripped-cladding MMF-based refractometer are classed according to 3 conditions or RI zones. Zone I is defined for the condition when the light is fully guided by TIR in the sensing region and, therefore, only EWA occurs as the unique sensing mechanism. This is illustrated in Fig. 2.

Zone I is then defined when $\theta_{b}>\theta_{c}$, which corresponds to $n_{s m}$ $<n_{b}<n_{c l}$, where $n_{b}$ is expressed as 


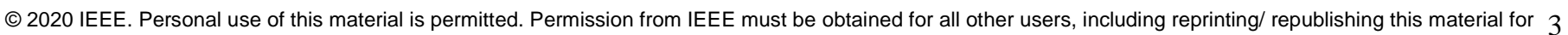
advertising or promotional purposes, creating new collective works for resale or redistribution to servers or lists, or reuse of any copyrighted components of this work in other works.

$$
n_{b}=n_{c o 2} \sin \theta_{b}
$$

The guided optical power, $\left(P_{L}\right)_{I}$, along the MMF for a sensing region of length $L$, can then be estimated by integrating the incident optical power for each ray $\left(\theta_{i}\right)$ from $\theta_{b}$ to $90^{\circ}$ through [25]

$$
\left(P_{L}\right)_{I}=\int_{\theta_{b}}^{90^{\circ}} P_{0}\left(\theta_{i}\right) e^{\left(-N\left(\theta_{i}\right) T\left(\theta_{i}\right) L\right)} d \theta_{i}
$$

where $P_{0}$ represents the input angular power density into the MMF while $N\left(\theta_{i}\right)$ is the number of reflections per unit length for an incident ray with angle $\theta_{i}$ and $T$ is the transmission coefficient of the evanescent waves (EWs) penetrating into the external sensing medium, respectively. These two parameters can be given by [32]

$$
\begin{gathered}
N\left(\theta_{i}\right)=\frac{\cot \theta_{i}}{2 a_{2}} \\
T\left(\theta_{i}\right)=\frac{\alpha \lambda n_{c o 2} \cos \theta_{i}}{\pi n_{s m}{ }^{2} \cos ^{2} \theta_{c s m} \sqrt{\cos ^{2} \theta_{c s m}-\cos ^{2} \theta_{i}}}
\end{gathered}
$$

Here, $\alpha$ is defined as the absorption coefficient of the sensing medium, and $\theta_{c s m}$ the equivalent critical angle at the interface core-sensing medium $\left(\theta_{c s m}=\sin ^{-1}\left(n_{s m} / n_{c o 2}\right)\right)$. Note that $N\left(\theta_{i}\right) L$ is the total number of reflections and is hence rounded off to the nearest integer number during computing.

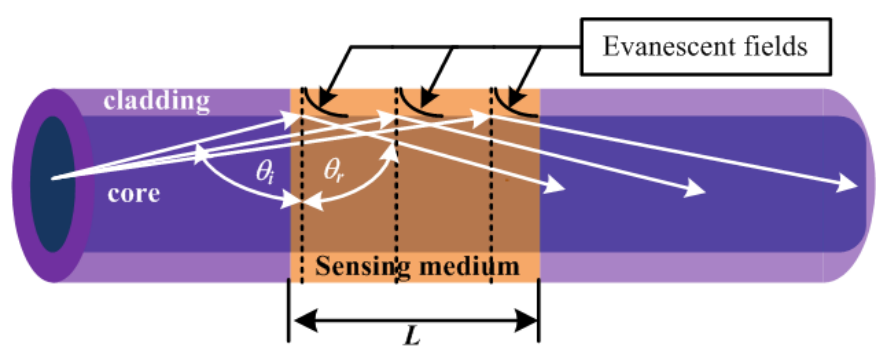

Fig 2: Sensing mechanism in Zone I based uniquely on EWA.

A clearer and more intuitive illustration of the EWA sensing mechanism in Zone I is shown in Fig. 3. The results physically demonstrate the evolution of EWs as a function of RI values. In this work, 2D wave optics simulation in the COMSOL Multiphysics environment is used since simulation in 3D would require excessively high computational load due to extremely high number of meshes to be generated. In addition, 3D simulation is not required due to the cylindrical symmetry of the MMF structure. During simulation, the core diameter used is proportionally reduced to $60 \mu \mathrm{m}$ instead of $200 \mu \mathrm{m}$ to avoid unwanted overloading during the computation. Further, as illustrated in Figs. 3(a) and 3(b), where for $\theta_{b}>\theta_{c s m}$, the beam from the lead-in SMF is propagated entirely in the MMF by TIR. The penetration of EWs into the functional cladding (FC) representing the sensing medium thus increases with increasing $n_{s m}$ as illustrated in Fig. 3(c). This can be clearly understood from (9), as, for a given $\theta_{i}$, the square root term of the denominator decreases towards zero when $\theta_{c s m}$ increases, subsequently leading to an increase in $T$. For a sensing medium (or FC) index $n_{s m}$ of 1.435 RIU (inset in Fig. 3(b)), more EW power (higher-intensity blue shade in FC) is observed in the FC area compared to that for a lower $n_{s m}$ of $1.385 \mathrm{RIU}$ (see inset of Fig. 3(a)). This will consequently contribute to decreasing optical power being guided along the fiber since more EW power is absorbed in the FC.

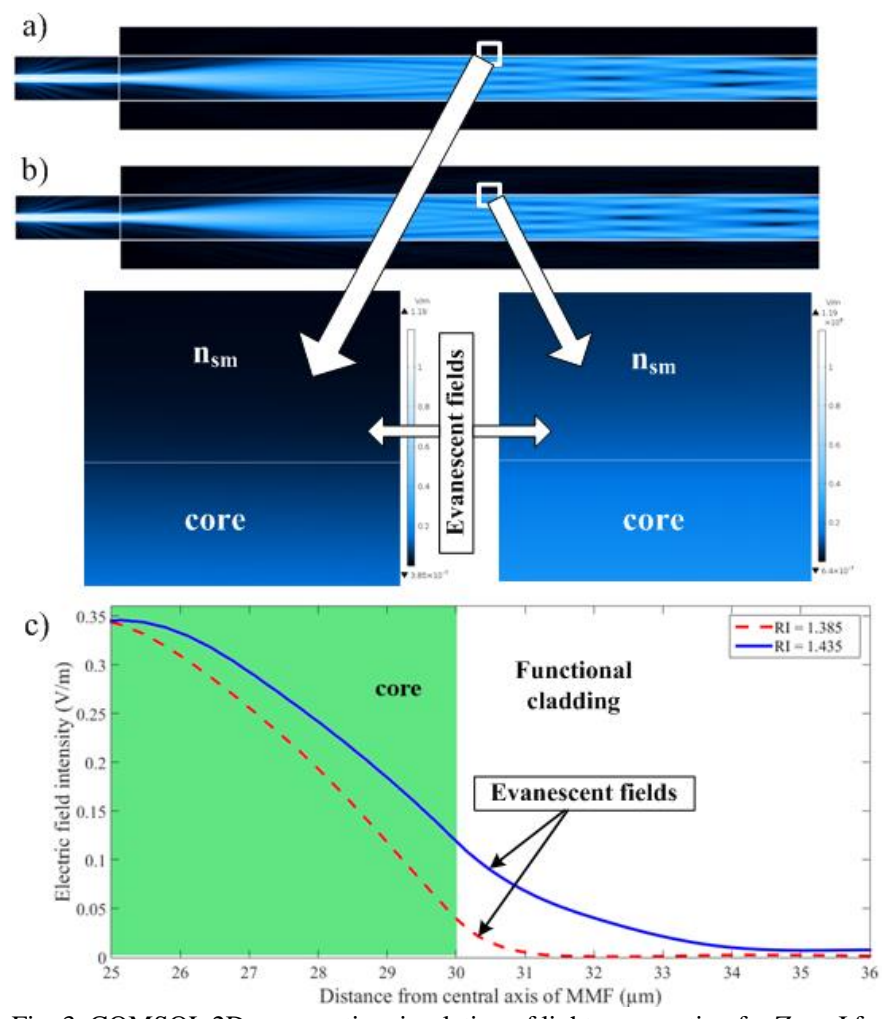

Fig. 3: COMSOL 2D wave optics simulation of light propagation for Zone I for (a) $n_{s m}=1.385$, (b) $n_{s m}=1.435$, and (c) evanescent waves penetrating into FC via exponential decay of the electric fields without any mode loss to FC or external medium.

In the case of the second condition or Zone II, where $n_{s m}>n_{b}$ but $n_{s m}<n_{c o 2}$, a different sensing mechanism occurs. Here, in parallel to EWA, there is an additional contribution from mode losses due to the increase in critical angle in the MMF sensing region (see Fig. 4). This is proportional to an increase in $n_{s m}$ and will induce a larger number of modes being transmitted or refracted into the external medium, hence leading to increasing mode losses. Nevertheless, there are also Fresnel reflections from rays with individual incident ray angle $\theta_{i}<\theta_{c s m}$ which are no longer guided by TIR. Subsequently, the model for Zone II can be described by

$$
\left(P_{L}\right)_{I I}=\int_{\theta_{\mathrm{csm}}}^{90^{\circ}} P_{0}\left(\theta_{i}\right) e^{\left(-N\left(\theta_{i}\right) T\left(\theta_{i}\right) L\right)} d \theta_{i}+\int_{\theta_{b}}^{\theta_{c \mathrm{~cm} m}} P_{0}\left(\theta_{i}\right) R\left(\theta_{i}\right)^{N\left(\theta_{i}\right) L} d \theta_{i}
$$

The first term on the right of (10) is the usual EWA contribution from the TIR guided modes which still exist, while the second term corresponds to the influence of Fresnel reflections for rays from $\theta_{b}$ to $\theta_{c s m}$ which are refracted to the exterior. $R$ represents the reflectivity and can be calculated as a function of the reflectance under both $p$-polarization $\left(r_{p}\right)$ and $s$-polarization $\left(r_{\mathrm{s}}\right)$, respectively, as [33]

$$
R\left(\theta_{i}\right)=\frac{1}{2}\left(r_{p}\left(\theta_{i}\right)^{2}+r_{s}\left(\theta_{i}\right)^{2}\right)
$$




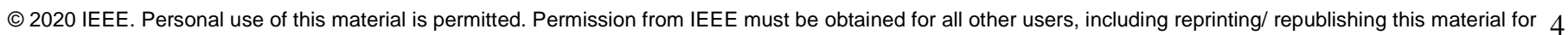
advertising or promotional purposes, creating new collective works for resale or redistribution to servers or lists, or reuse of any copyrighted components of this work in other works.

for which the reflectance under the respective polarizations is given by

$$
r_{p}\left(\theta_{i}\right)=\frac{n_{s m} \cos \theta_{i}-n_{c o 2} \cos \theta_{t}}{n_{s m} \cos \theta_{i}+n_{c o 2} \cos \theta_{t}} ; \quad r_{s}\left(\theta_{i}\right)=\frac{n_{c o 2} \cos \theta_{i}-n_{s m} \cos \theta_{t}}{n_{c o 2} \cos \theta_{i}+n_{s m} \cos \theta_{t}}
$$

However, the contribution from Fresnel reflections in Zone II is very weak (of the order of $\sim 10^{-6}$ to $10^{-5}$ ) since the index contrast between $n_{c o 2}$ and $n_{s m}$ is relatively small $\left(n_{c o 2}-n_{b} \cong\right.$ 0.0089 RIU). Hence, these reflections can be neglected. Subsequently, the model for Zone II in (10) can be simplified to a combination of EWA for rays which are still guided by TIR, similar to Zone I, and mode losses for rays from $\theta_{b}$ to $\theta_{c s m}$ which no longer exist in the integral given in (13) below. This is illustrated in Fig. 4.

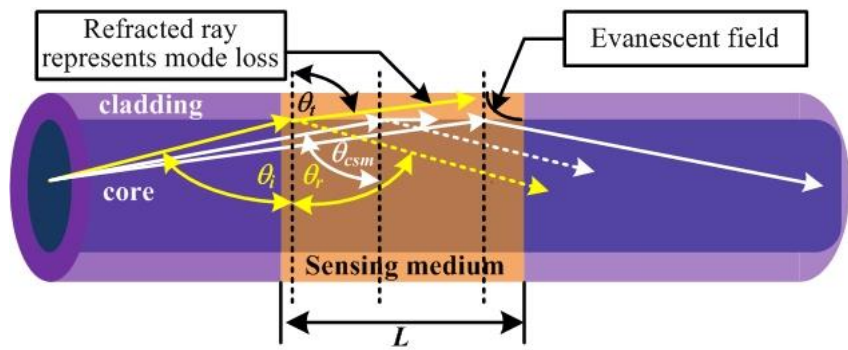

Fig. 4: Illustration of EWA and mode losses represented by the yellow rays due to modification of $\theta_{c s m}$ with respect to $n_{s m}$ in Zone II.

The input rays subject to EWA are now thus considered from $\theta_{c s m}$ to $90^{\circ}$, and can be expressed by

$$
\left(P_{L}\right)_{I I}=\int_{\theta_{c s m}}^{90^{\circ}} P_{0}\left(\theta_{i}\right) e^{\left(-N\left(\theta_{i}\right) T\left(\theta_{i}\right) L\right)} d \theta_{i}
$$

Finally, for Zone III, in which the sensing region of the refractometer is employed for measuring $n_{s m}>n_{c o 2}$, propagation by TIR is no longer supported. Nevertheless, some optical power will still be guided by the phenomenon of external reflection while the majority of the rays are refracted and transmitted into the external medium (or FC). This can be explained using Fresnel equations to describe the interaction of the optical rays at the interface of two media with different RI values, as illustrated in Fig. 5.

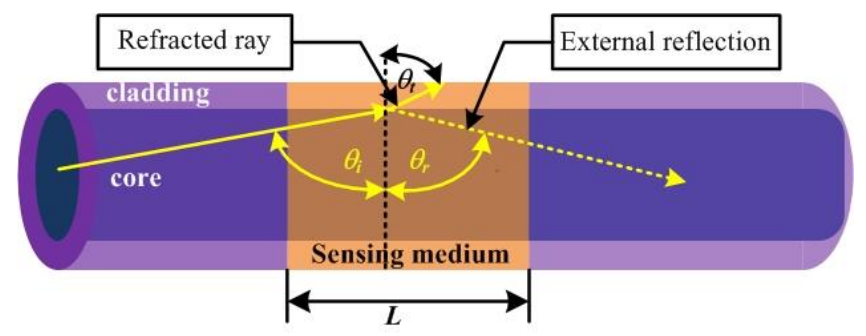

Fig. 5: Illustration of sensing mechanism in Zone III by Fresnel reflection.

When $n_{s m}>n_{c o 2}$, the optical power from the core will principally be transmitted to the external region and lost. There is, however, a small amount of power being reflected back into the core through Fresnel reflections in (11) and (12), whose guided power can be calculated via

$$
\left(P_{L}\right)_{I I I}=\int_{\theta_{b}}^{90^{\circ}} P_{0}\left(\theta_{i}\right) R\left(\theta_{i}\right)^{N\left(\theta_{i}\right) L} d \theta_{i}
$$

\section{Experimental investigation and validation}

The refractometer operates on the basis of ratiometric intensity measurements through a differential probe configuration, with one SMF-MMF serving as the sensing arm, and the other the reference arm to compensate common-mode noises, as shown in Fig. 6. The emitted beam from a $1550 \mathrm{~nm}$ SM fiber-pigtailed DFB laser diode is transmitted via a 50/50 SM fiber coupler to obtain two equal interrogating beams. Since SMF is directly spliced to MMF (Thorlabs FP200ERT) [35] with $N A_{2}=0.50$, the injected beam will be diffracted at the SMF-MMF interface, then guided into and along the latter fiber. Two identical $G e$-type Thorlabs PDA50B photodetectors are used for measuring the guided optical powers at the MMF outputs. The measured data are next recorded by a dedicated 1.25 MHz National Instrument data acquisition system (DAQ)

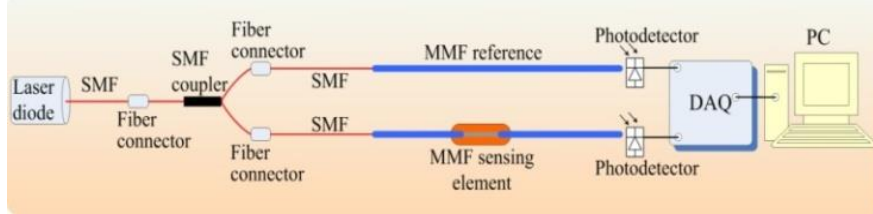

for further processing.

Fig. 6: Experimental schematic of hybrid differential SMF-MMF refractometer for RI measurement with common-mode noise compensation.

A combination of water-glycerol mixture with different concentrations is employed as the sensing medium to obtain an RI range from 1.3164 to $1.4571 \mathrm{RIU}$ at $1550 \mathrm{~nm}$, similar to [36]. The corresponding absorption coefficient $\alpha_{s m}$ is dependent on the experimentally determined extinction coefficient, calculated according to the procedures in [25,36-38]. For inducing RI values beyond those of glycerol (i.e. for RI > 1.4571), calibrated oils from Cargille Laboratories ranging from 1.468 to 1.608 RIU are employed.

\section{RESULTS AND DISCUSSION}

FEM-based simulation cannot be used to support 3D MMF simulation, since its dimension with respect to the operating wavelength will contribute to a very high mesh number and, thus, computational load. Hence, the most effective simulation technique to estimate the response of this refractometer is by combining the models through Gaussian beam analysis, ray optic principles, and analytical wave optics.

Equation (1) is first used to calculate $\theta_{b}\left(\theta_{b}=90^{\circ}-\beta_{b}=83.6^{\circ}\right)$, before obtaining the $z_{i}$-distance through (3), giving $z_{i}=896.94$ $\mu \mathrm{m} . w_{z}$ is next calculated using (2) for the SMF's $w_{0}\left(w_{0}=4.85\right.$ $\mu \mathrm{m})$, given as $0.5 \mathrm{MFD} \mathrm{SMF}_{\mathrm{SM}}$. Subsequently, using (4) and (5), the optical intensity and power at $z_{i}$ can be obtained.

Inferring from the sensing principles, the boundary between Zone I and Zone II will be at $n_{b}$, an index corresponding to $\theta_{b}$, due to $N A_{2}(\mathrm{MMF})$ being higher than $N A_{1}$ (SMF). From (6), this boundary is determined to be at $n_{b} \sim 1.4351$ RIU, and, consequently, the beam will be entirely guided by TIR for $n_{s m}$ $<n_{b}$. Additionally, to estimate the remaining guided power by 
TIR in Zone I, as expressed by (7), the input angular power

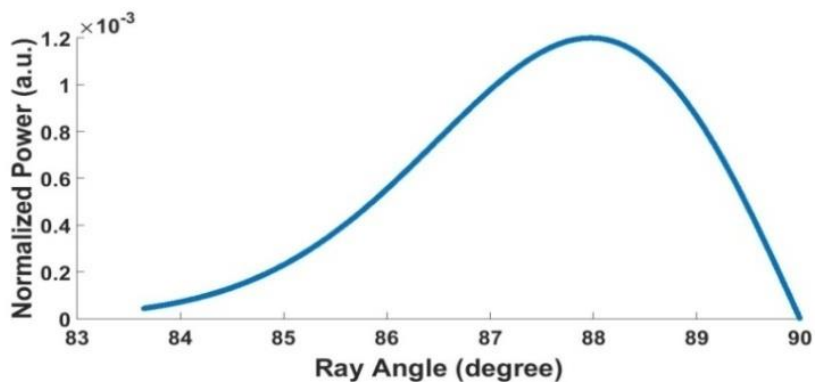

density distribution $P_{0}\left(\theta_{i}\right)$ in Fig. 7 for each angle has to be known. This is obtained using the method proposed in earlier work [25], for $P_{0}\left(\theta_{i}\right)$ from $\theta_{b}$ to $90^{\circ}$.

Fig. 7: Input angular power density distribution $P_{0}\left(\theta_{i}\right)$ injected into MMF for incident angles from $\theta_{b}$ to $90^{\circ}$.

Hence, by substituting the values of $P_{0}\left(\theta_{i}\right)$ for all incident rays (from $\theta_{b}$ to $90^{\circ}$ ) into (7)-(9), the guided power under any perturbation in Zone I (i.e. $n_{s m}<1.4351$ (or $n_{b}$ )) can thus be accurately estimated. On the other hand, for $n_{s m}>1.4351$ and up to 1.444 (or $n_{c o 2}$ ), the Zone II sensing response is obtained by numerically integrating $P_{0}\left(\theta_{i}\right)$ from $\theta_{c s m}$ to $90^{\circ}$ with (13). Finally, for Zone III, the guided power can be estimated by employing (11), (12), and (14) for all incident rays from $\theta_{b}$ to $90^{\circ}$.

The complete simulation results of the RI response across the 3 zones are plotted and corroborated experimentally in Fig. 8 for two sensing lengths of $2 \mathrm{~cm}$ and $5 \mathrm{~cm} \pm 0.1 \mathrm{~cm}$. The response curves show that, in comparison with previous results [25], the dynamic range of Zone I is wider in this work (from 1.3164 to $1.4351 \mathrm{RIU})$, contrary to Zone II, due to the injected $\theta_{b}$ from the lead-in SMF into the MMF being higher (close to $90^{\circ}$ ).

For Zone I response, a higher power loss is achieved for the longer sensor $(5 \mathrm{~cm})$, and the measured optical power decreases for increasing RI due to increasing EWA as predicted by (7). Higher power losses are also achieved by the 5-cm sensor in Zone II since in this zone, there is still EWA which additionally contributes to the response. Furthermore, the sharp decrease in the Zone II response for both the $2-\mathrm{cm}$ and the $5-\mathrm{cm}$ sensors results from the mode losses due to the modification of the critical angle for increasing RI values. This leads to a higher number of modes being continuously lost through refraction. The dynamic range for Zone II response undergoes no change compared with previous results [25] since the characteristics of the MMF core are identical, i.e. PCS MMF with a silica core. However, the power response significantly increases comparatively [25], in particular for the shorter 2-cm sensor since the incident beam angle from the lead-in SMF into the MMF, $\theta_{b}$, is closer to $90^{\circ}$. Hence, the total number of reflections along the sensor decreases, resulting in more power being guided along the MMF, according to (14).

The power response curves are next derived based on the models, as a function of RI across all 3 zones, to obtain the sensitivities for both sensors which are plotted in Fig. 9. Very high sensitivities are achieved in Zone II for both sensors, with the best sensitivity (-248 a.u./RIU, which is equivalent to 2480 V/RIU, depending on the photodetector system employed) achieved by the 2-cm stripped-cladding MMF. This can be justified by the sharp decrease in the optical power response in Fig. 8 as the shorter sensor is subject to the least EWA. Consequently, it suffers higher losses through the modification of the critical angle (mode loss mechanism) as previously explained in [25]. As the measured index approaches $n_{c o 2}$ at the end of Zone II, the sensitivity lessens. The optical power (Fig. 8) then exhibits a flat response at the exact boundary between Zone II and Zone III, consequently resulting in a zerosensitivity value. The sensitivity at the beginning of Zone I, on the contrary, is very low (-0.016 a.u./RIU and -0.040 a.u./RIU for the $2-\mathrm{cm}$ and $5-\mathrm{cm}$ sensors, respectively) since the slow gradient of the power response in this zone is affected by the low penetration of EWs into the cladding and a lower total number of reflections $(N L)$ along the decladded area. This is due to $\theta_{b}$ in MMF being close to $90^{\circ}\left(\sim 83.6^{\circ}\right)$. Consequently, a higher sensitivity for Zone I is achieved by the longer sensing region $(5 \mathrm{~cm})$ due to stronger EWA from the contribution of higher $N L$ in this sensor. Further, the sensitivity $(S)$ curves in Zone III (Fig. 9) describe a higher sensitivity being achieved for the 2-cm sensor. However, this sensitivity then decreases for further increasing RI. Nevertheless, the sensitivities for both the $2-\mathrm{cm}$ and $5-\mathrm{cm}$ sensors in Zone III are higher (11.82 a.u./RIU and 4.99 a.u./RIU, respectively) compared to previous results [25] and even in comparison with the shortest $(1 \mathrm{~cm})$ of the previous sensors where a sensitivity of only 1.20 a.u./RIU was obtained for this zone. This is due to a lower number of reflections, which contribute to higher transmitted power as well as a higher gradient which represent the sensor sensitivity. 
(C) 2020 IEEE. Personal use of this material is permitted. Permission from IEEE must be obtained for all other users, including reprinting/ republishing this material for 6 advertising or promotional purposes, creating new collective works for resale or redistribution to servers or lists, or reuse of any copyrighted components of this work in other works.

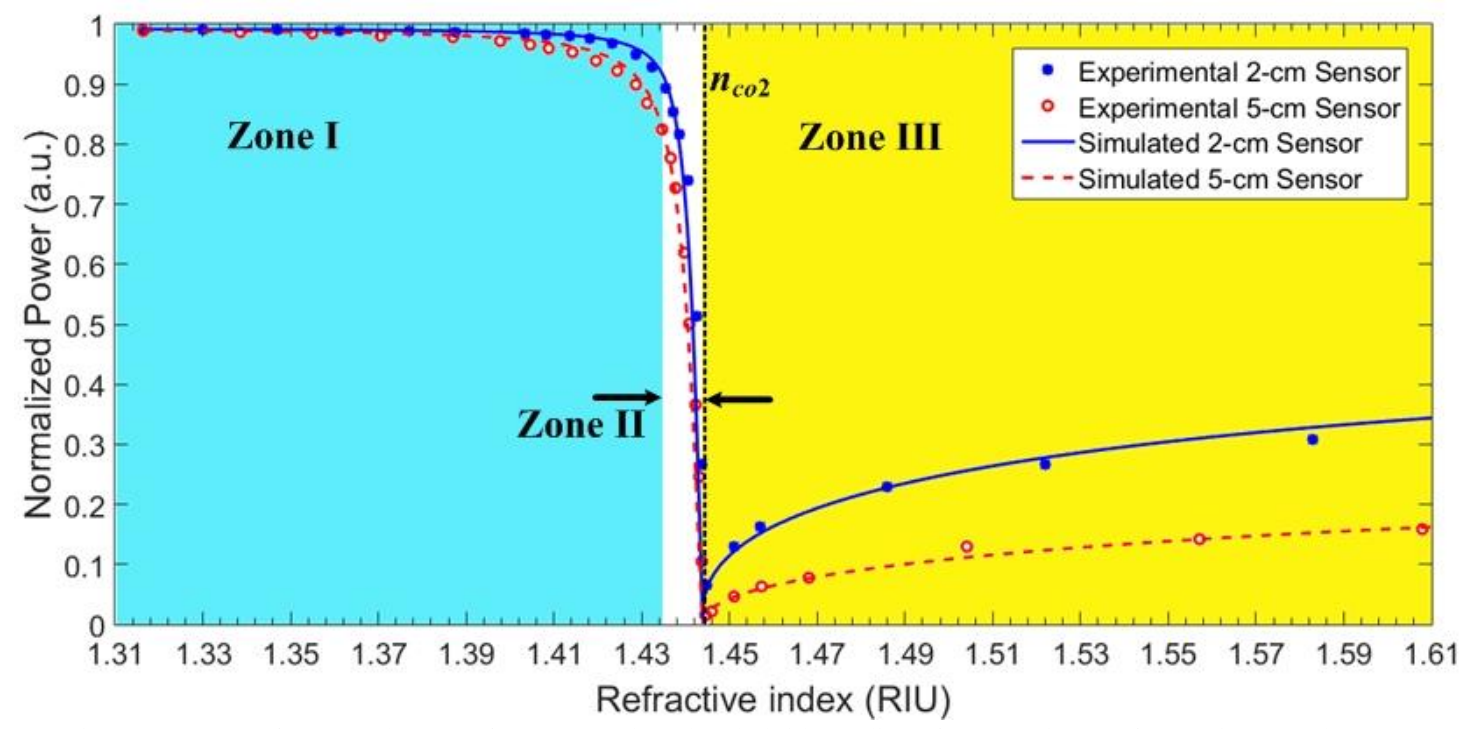

Fig. 8: Response of 2-cm and 5-cm refractometers based on hybrid coupling of SMF and MMF for varying RI values.
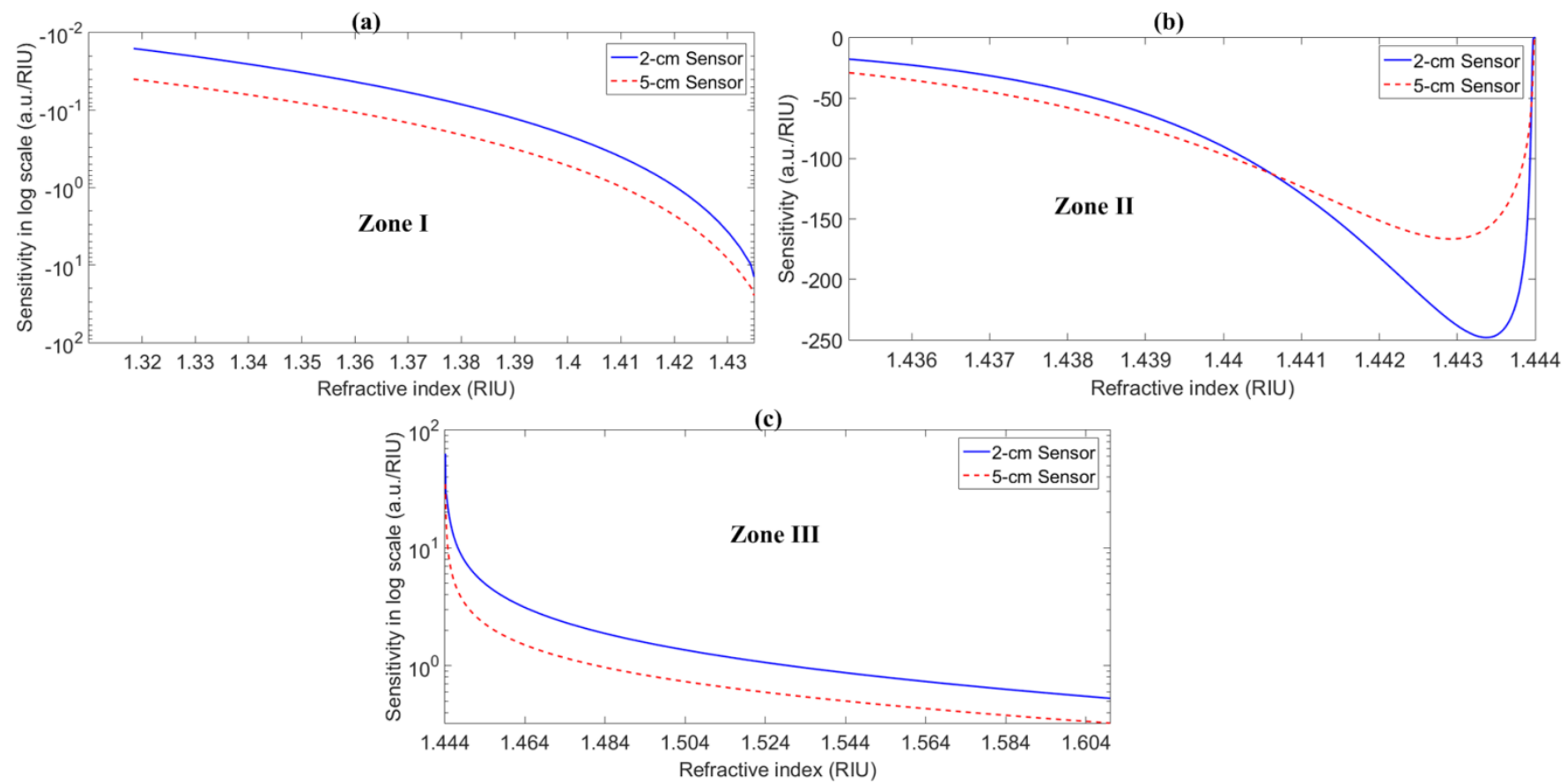

Fig. 9: Sensitivity curves of 2-cm and 5-cm SMF-MMF hybrid refractometers, (a) in Zone I, (b) in Zone II, and (c) in Zone III. 


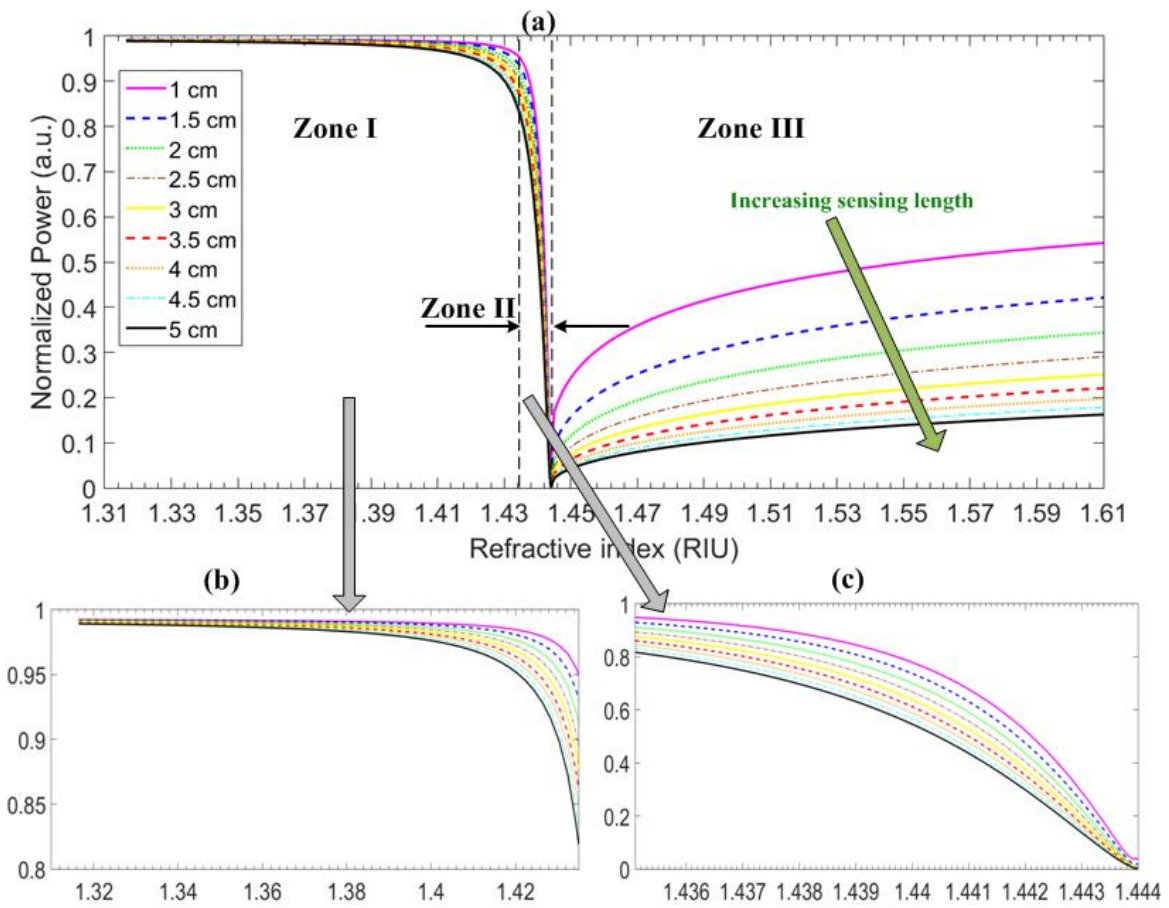

Fig. 11: Simulation results of hybrid MMF refractometers for sensing length from $1 \mathrm{~cm}$ to $5 \mathrm{~cm}$, with (a) response for Zones I, II and III, (b) zoom for Zone I, and (c) zoom for Zone II.
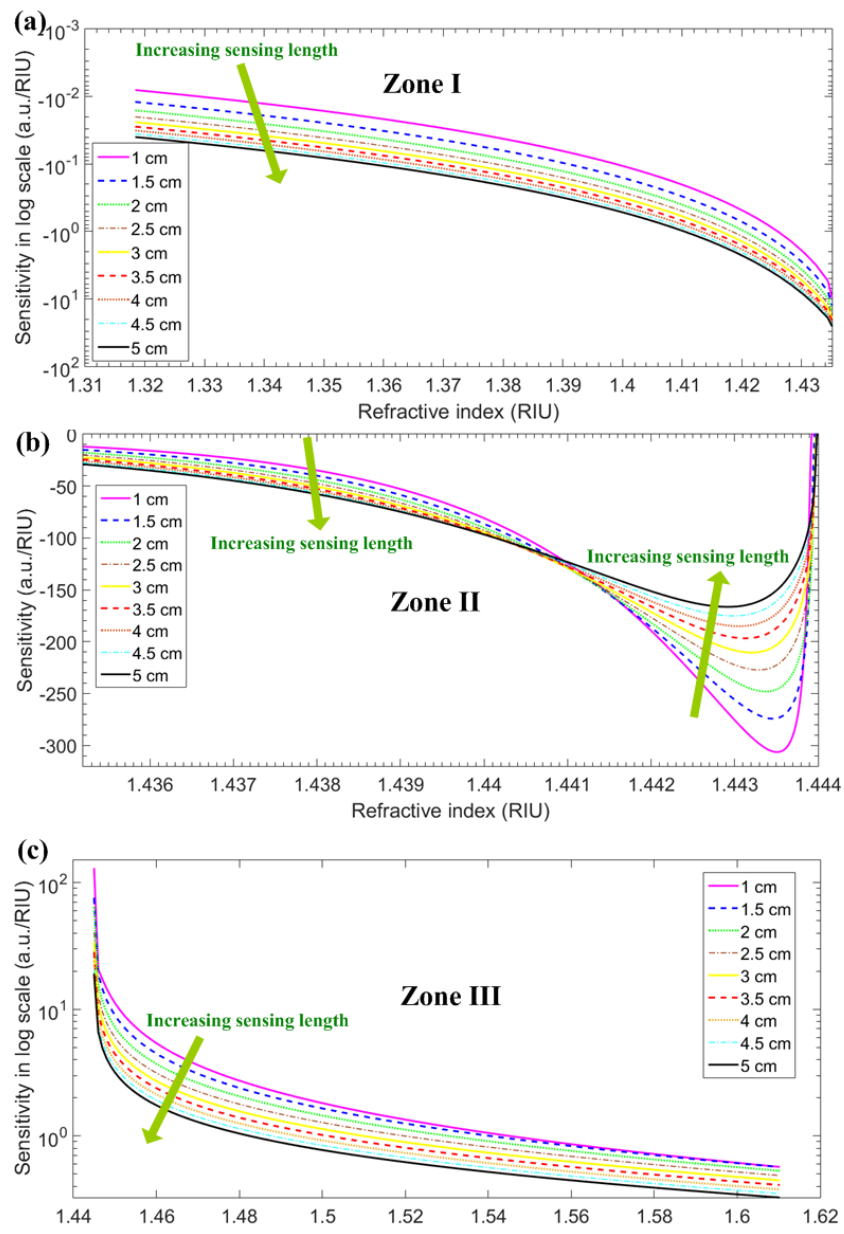

Fig. 10: Sensor sensitivities for, (a) Zone I, (b) Zone II, and (c) Zone III.
The refractometer resolution, Res, is next determined using the sensitivity curves in Fig. 9 by taking 6 times the RMS measurement noise, $\sigma$, corresponding to $\sim 99.7 \%$ confidence level, divided by the sensitivity, i.e. Res $=6 \sigma / S$ [39]. Results from this work are first compared against our previous benchmark in [25]. The best resolution obtained here is $2.76 \times 10^{-6} \mathrm{RIU}$, achieved by the $2-\mathrm{cm}$ sensor in Zone II. This is an 8-time improvement over the previous refractometer where the best resolution was $2.245 \times 10^{-5} \mathrm{RIU}$ for the $1-\mathrm{cm}$ sensor. A higher resolution is achieved in this work, due to a narrower input angular power density (from $83.6^{\circ}$ to $90^{\circ}$ ) that thus allows to achieve both a higher sensitivity and a sharper response in Zone II. Further, the highest resolution obtained for Zone III in this work is $5.30 \times 10^{-5} \mathrm{RIU}$, also achieved by the $2-\mathrm{cm}$ sensor, as compared to $1.39 \times 10^{-4} \mathrm{RIU}$ for the $5-\mathrm{cm}$ sensor. These values are at least one order of magnitude better than that obtained $\left(1.45 \times 10^{-3} \mathrm{RIU}\right)$ previously for the shortest sensor $(1 \mathrm{~cm})$ in Zone III, in direct accordance with a higher sensitivity due to a lower number of reflections obtained in the sensing region. However, the resolution achieved at the beginning of Zone I is limited to $1.63 \times 10^{-2}$ RIU and $3.39 \times 10^{-2}$ RIU for the $2-\mathrm{cm}$ and 5 -cm sensors, respectively. These are relatively weak compared to the best resolution $\left(8.34 \times 10^{-3}\right.$ RIU) obtained from the previous $4-\mathrm{cm}$ long refractometer due to less EWA being achieved by the sensing region in this work.

Although the models developed in this work are principally used to accurately estimate the performance (in terms of response, resolution/sensitivity, RI delimitation, etc) of the hybrid SMF-MMF refractometers, the capability of these analytical models can also be extended to optimize the future design of other similar fiber refractometers. For example, in 


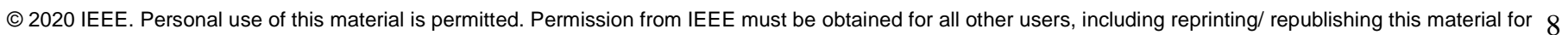
advertising or promotional purposes, creating new collective works for resale or redistribution to servers or lists, or reuse of any copyrighted components of this work in other works.

Fig. 10, we investigate the influence of the sensing length on the response of the refractometers for lengths ranging from 1 $\mathrm{cm}$ to $5 \mathrm{~cm}$. The simulation results in Fig. 10 demonstrate that the initial response of the refractometers under study (from 1 $\mathrm{cm}$ to $5 \mathrm{~cm}$ ) in Zones I and II are relatively difficult to distinguish at low RI values. Nevertheless, in Zone I, the respective sensor response then separates with increasing RI since more optical power is exponentially absorbed via EWA with increasing sensing length. With regard to Zone II, the response of the refractometers subsequently converges when the measured RI increases. This is due to the decreasing influence of EWs since more modes are exponentially lost (or refracted) into the sensing medium. On the other hand, the response of each refractometer in Zone III can be clearly distinguished since the optical power responses as a power of $L$ and $N$ as described by (14).

Figure 11 displays the sensitivity values for refractometers with $1 \mathrm{~cm}$ to $5 \mathrm{~cm}$ of sensing length. The sensitivity curves of these sensors in Zone I converge with increasing RI to a crossing point in Zone II. Before this crossing point, the longer sensor has the largest sensitivity as we have seen and explained in Fig. 9. After the crossing point, the sensitivity of the longest sensor decreases in sensitivity while that of the shortest sensor becomes increasingly sensitive. This is due to the sharper response of the shorter sensing elements after the 1.4410 RIU point. In Zone III, the sensitivity curves decrease significantly and very rapidly beyond 1.445 RIU. However, the sensitivities of the shorter sensing elements are always larger than those of the longer sensors.

As shown earlier in this paper, the present model is highly accurate and, hence, can additionally be used to design a hybrid SMF-MMF RI sensor with the desired sensitivity. For instance, using Fig.11 as the baseline (more detailed abacus generated with our model could also be used), the maximum length required to design a sensor with a sensitivity of at least 300 a.u./RIU can be determined. In this example, the maximum length of the sensing region must be $1 \mathrm{~cm}$. Nonetheless, the model, which is relatively fast to run, could be coupled with an optimization method to automatically analyze the sensor parameters and find the correct length of the sensing region.

\section{CONCLUSION}

Two hybrid SMF-MMF fusion splice-coupled refractometers have been realized to significantly improve the sensitivity in the Zone II and Zone III RI regimes by a factor of 8 to 10 compared to previous work [25] while concurrently reducing parasite mechanical perturbations advantageously. Since the injected beam angle from the lead-in SMF corresponds to its NA, this then determines the equivalent angle to the RI value at the Zone I and Zone II boundary. Accordingly, the ray power distribution density which propagates along the MMF can theoretically be obtained by using Gaussian beam analysis for incident beam angles from $\theta_{b}\left(83.6^{\circ}\right)$ to $90^{\circ}$, which are obtained from the value of $\mathrm{NA}_{1}$. Subsequently, the optical power response for both the $2-\mathrm{cm}$ and $5-\mathrm{cm}$ refractometers can be estimated by integrating the ray power distribution density into the models for Zone I and Zone II through ray optic principles and the analytical equation for evanescent waves. The model for Zone III, on the other hand, employs the ray power distribution density combined with Fresnel equations.

The results for the three sensing zones are then corroborated experimentally by decladding two MMFs over $2 \mathrm{~cm}$ and $5 \mathrm{~cm}$ in length, respectively, demonstrating very high agreement with the simulation results. In addition, since the incident beam angle $\left(\theta_{b}\right)$ from the lead-in SMF is close to $90^{\circ}$, the dynamic range of Zone II is consequently very narrow (from 1.4351 to 1.444 RIU), compared to the relatively wider range obtained in Zone I. In terms of sensitivity, the results achieved with these hybrid SMF-MMF splice-coupled refractometers show significantly improved sensing performance by more than 8 times, in particular for the shorter $2-\mathrm{cm}$ sensor over Zones II and III, respectively, compared to our previous results [25]. On the other hand, the sensitivity of these sensors in Zone I, in particular at the beginning of this zone, is very low $(-0.016$ a.u./RIU and -0.034 a.u./RIU for the $2-\mathrm{cm}$ and $5-\mathrm{cm}$ sensors, respectively) when compared to -0.392 a.u./RIU previously achieved. This, however, increases exponentially with increasing RI toward the boundary between Zone I and Zone II, in accordance with the model for Zone I described in (7).

Using the proposed models enables us to estimate the performance of MMF refractometers with different sensing lengths with relative ease since the simulation time for each sensing length is less than 20 seconds. Hence, various lengths can be efficiently and accurately simulated to obtain the desired sensor performance.

\section{ACKNOWLEDGMENT}

H. Apriyanto gratefully acknowledges support from the Indonesia Endowment Fund for Education (LPDP), Ministry of Finance, Republic of Indonesia, in the form of a $\mathrm{PhD}$ scholarship. H. C. Seat also acknowledges support from Aquitaine Electronique through an industrial secondment. The authors would like to thank C. Tronche and F. Jayat for technical support during the experimental set-up. This work was part-supported by the Agence Nationale de la Recherche (grant number ANR-13-IS06-0001-04).

\section{REFERENCES}

[1] W. B. Jensen, "Refractometers," in Oesper Museum Booklets on the History of Chemical Apparatus No. 3, University of Cincinnati, 2014.

[2] J. O. W. Norris, "Optical fiber chemical sensors: fundamentals and applications," in Optical Fiber Sensor Technology, K. T. V. Grattan \& B. T. Meggitt, Eds., Boston, MA, USA: Springer, 2000, pp. 337-375.

[3] K. T. V. Grattan and T. Sun, "Fiber optic sensor technology: an overview," Sens. Actuators A Phys., vol. 82, pp. 40-61, May 2000.

[4] A. D. Kersey, "A review of recent developments in fiber optic sensor technology," Opt. Fiber Technol., vol. 2, pp. 291-317, Jul. 1996.

[5] B. Lee, "Review of the present status of optical fiber sensors," Opt. Fiber Technol. vol. 9, pp. 57-79, Apr. 2003

[6] O. Rodríguez-Quiroz, C. E. Domínguez-Flores, D. Monzón-Hernández, and C. Moreno-Hernández, "Hybrid fiber Fabry-Perot interferometer with improved refractometric response," J. Lightwave Technol., vol. 37, pp. 4268-4274, Sept. 2019.

[7] H. Fukano, T. Aiga and S. Taue, "High-sensitivity fiber-optic refractive index sensor based on multimode interference using small-core singlemode fiber for biosensing," Jpn. J. Appl. Phys., vol. 53, pp. 04EL08-104EL08-4, Mar. 2014. 
[8] M. d. C. Alonso-Murias, J. S. Velázquez-González and D. MonzónHernández, "SPR fiber tip sensor for the simultaneous measurement of refractive index, temperature, and level of a liquid," J. Lightwave Technol., vol. 37, pp. 4808-4814, Sept. 2019.

[9] J. Villatoro, D. Monzon-Hernandez, and D. Talavera, "High resolution refractive index sensing with cladded multimode tapered optical fibre," Elect. Lett., vol. 40, pp. 106-107, Jan. 2004.

[10] M. I. Zibaii, A. Kazemi, H. Latifi, M. K. Azar, S. M. Hosseini, and M. H. Ghezelaiagh, "Measuring bacterial growth by refractive index tapered fiberopticbiosensor," J. Photochem. Photobio. B Bio., vol. 101, 313-320 Dec. 2010.

[11] H. A. Rahman, S. W. Harun, M. Yasin, S. W. Phang, S. S. A. Damanhuri, $\mathrm{H}$. Arof, and H. Ahmad, "Tapered plastic multimode fiber sensor for salinity detection,” Sens. Actuators A Phys., vol. 171, pp. 219-222 Nov. 2011.

[12] C. B. Kim and C. B. Su, "Measurement of the refractive index of liquids at 1.3 and 1.5 micron using a fibre optic Fresnel ratio meter," Meas. Sci. Technol., vol. 15, pp. 1683-1686, Jul. 2004.

[13] P. Nath, H. K. Singh, P. Datta, K. C. Sarma, "All-fiber optic sensor for measurement of liquid refractive index," Sens. Actuators A Phys., vol. 148, pp. 16-18, Nov. 2008.

[14] W. Xu, X. G. Huang, and J. S. Pan, "Simple fiber-optic refractive index sensor based on Fresnel reflection and optical switch," IEEE Sensors J., vol. 13, pp. 1571-1574, May 2013. DOI: 10.1109/JSEN.2012.2236751.

[15] A. L. Chaudharia and A. D. Shaligramb, "Multi-wavelength optical fiber liquid refractometry based on intensity modulation," Sens. Actuators A Phys., vol.100, pp. 160-164, Sept. 2002.

[16] M. E. Muthuraju, A. R. Patlollaa, B. V. Canthadaia, and V. Pachavab, "Low cost fiber optic sensing of sugar solution," in Optical Fibers and Sensors for Medical Diagnostics and Treatment Applications XV, Proc. SPIE, vol. 9317, San Francisco, CA, USA, 2015, pp. 93170L.

[17] D.-J. Feng, M.-S. Zhang, G. Liu, Xi-L. Liu, and D.-F. Jia, "D-shaped plastic optical fiber sensor for testing refractive index," IEEE Sensors J., vol.14, pp. 1673-1676, May 2014. DOI: 10.1109/JSEN.2014.2301911.

[18] G. Liu and D. Feng, "Evanescent wave analysis and experimental realization of refractive index sensor based on D-shaped plastic optical fiber," Optik, vol. 127, pp. 690-693, Jan. 2016.

[19] A. Arie, R. Karoubi, Y. S. Gur, and M. Tur, "Measurement and analysis of light transmission through a modified cladding optical fiber with applications to sensors," Appl. Opt., vol. 25, pp. 1754-1758, 1986.

[20] A. Arie, M. Tur, and S. Goldsmith, "Measurement and analysis of a modified cladding optical fiber with various input illuminations," in Fiber Optic and Laser Sensors IV, Proc. SPIE, vol. 0718, Cambridge, MA, USA, 1987, pp. 160-167.

[21] A. Arie and M. Tur, "Light transmission through a modified cladding optical fiber - inverse problem analysis," in 6th Mtg in Israel on Optical Engineering, Proc. SPIE, vol. 1038, Tel Aviv, Israel, 1989, p. 225-232.

[22] C. F. Cole, A. J. Adams, and R. A. Sims, "Optical fiber refractometer for measuring the dispersion of turbid fluids," in Chemical, Biochemical, and Environmental Fiber Sensors IV, Proc. SPIE, vol. 1796, Boston, MA, USA, 1993, pp. 383-392.

[23] A. Mukherjee, D. Munsi, V. Saxena, R. Rajput, P. Tewari, V. Singh, A. K. Ghosh, J. John, H. Wanare, and P. Gupta-Bhaya, "Characterization of a fiber optic liquid refractive index sensor," Sens. Actuators B Chem. vol. 145, pp. 265-271, Mar. 2010.

[24] H. Apriyanto, G. Ravet, O. D. Bernal, M. Cattoen, F. Lizion, and H. C. Seat, "A multimode fiber refractive index sensor," in Proc. IEEE Sensors 2016, Orlando, FL, USA, 2016, pp. 277-279.

[25] H. Apriyanto, G. Ravet, O. D. Bernal, M. Cattoen, H. C. Seat, V. Chavagnac, F. Surre, J. H. Sharp, "Comprehensive modeling of multimode fiber sensors for refractive index measurement and experimental validation," Sci. Rep., vol. 8:5912, Apr. 2018.

[26] N. S. Kapany and J. N. Pike, "Fiber Optics. Part IV. A Photorefractometer," J. Opt. Soc. Am., vol. 47, pp. 1109-1117, 1957.

[27] N. S. Kapany and D. A. Pontarelli, "Photorefractometer I. Extension of Sensitivity and Range," Appl. Opt., vol. 2, pp. 425-430, 1963.

[28] N. S. Kapany and D. A. Pontarelli, "Photorefractometer II. Measurement of N and K," Appl. Opt., vol. 2, pp. 1043-1048, 1963.

[29] A. E. Siegman, Lasers, Sausalito, CA, USA: University Science Books, 1986.

[30] A.M. Kowalevicz, Jr. and F. Bucholtz, "Beam Divergence from an SMF28 Optical Fiber," Naval Research Laboratory, Washington, DC, USA, Memo. Rep. NRL/MR/5650--06-8996, Oct. 2006.

[31] https://www.colorado.edu/physics/phys4510/phys4510_fa05/Chapter5. pdf
[32] B. K. Keller, M. D. DeGrandpre, and C. P. Palmer, "Waveguiding properties of fiber-optic capillaries for chemical sensing applications," Sens. Actuators B Chem., vol. 125, pp. 360-371, Aug. 2007.

[33] B. E. A. Saleh and M. C. Teich, Fundamental of Photonics, 2nd ed., Hoboken, NJ, USA: Wiley, 2007

[34] https://www.comsol.com/blogs/using-perfectly-matched-layers-andscattering-boundary-conditions-for-wave-electromagnetics-problems/ https://www.thorlabs.com/drawings/770825baa935cad0-C8F3C0D5A4B3-20D8-0033E053A3FD8E10/FP200ERT-SpecSheet.pdf

[36] J. E. Saunders, C. Sanders, H. Chen, and H. P. Loock, "The refractive index of common solvents and solutions at $1550 \mathrm{~nm}$," Appl. Opt., vol. 55, pp. 947-953, 2016.

[37] A. A. Riziq, C. Erlick, E. Dinar, and Y. Rudich, "Optical properties of absorbing and non-absorbing aerosols retrieved by cavity ting down (CRD) spectroscopy," Atmos. Chem. Phys., vol. 7, pp. 1523-1536, Mar. 2007.

[38] F. A. Jenkins, and H. E. White, Fundamental of Optics, pp. 472-473, New York, USA: McGraw-Hill, 1950

[39] A. J. Fleming, "Review of nanometer resolution position sensors: Operation and performance," Sens. Actuators A Phys., vol. 190, pp. 106-126, Feb. 2013. 